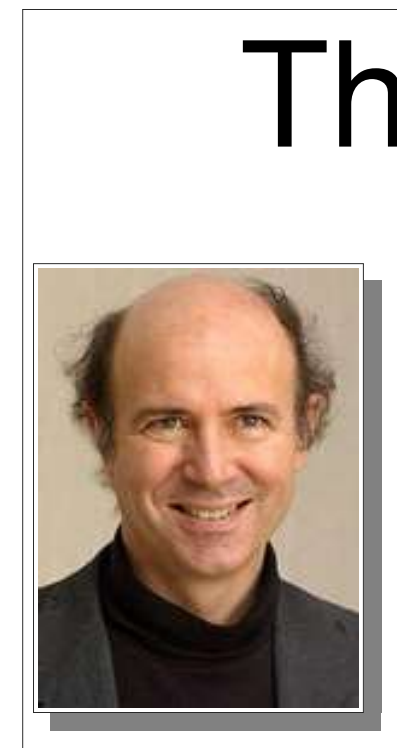

\title{
The Origin of Mass
}

Frank Wilczek

that occasionally this sophisticated work gives answers to childlike questions about familiar things. Here l'd like to describe how myown work on subnuclear forces, the world of quarks and gluons, casts brilliant new light on one such child-like question: What is the origin of mass?

\section{Has Mass an Origin?}

That a question makes grammatical sense does not guarantee that it is answerable, or even coherent.

The concept of mass is one of the first things we discuss in my freshman mechanics class. Classical mechanics is, literally, unthinkable without it. Newton's second law of motion says that the acceleration of a body is given by dividing the force acting upon it by its mass. So a bodywithout mass wouldn't know how to move, because you'd be dividing by zero. Also, in Newton's law of gravity, the mass of an object governs the strength of the force it exerts. One cannot build up an object that gravitates, out of material that does not, so you can't get rid of mass without getting rid of gravity. Finally, the most basic feature of mass in classical mechanics is that it is conserved. For example, when you bring together two bodies, the total mass is just the sum of the individual masses. This assumption is so deeply ingrained that it was not even explicitly formulated as a law. (Though I teach it as Newton's Zeroth Law.) Altogether, in the Newtonian framework it is difficult to imagine what would constitute an "origin of mass," or even what this phrase could possibly mean. In that framework mass just is what it is-a primary concept.

Later developments in physics make the concept of mass seem less irreducible. Einstein's famous equation $E=m c^{2}$ of special relativity theory, written in that way, betrays the prejudice that we should express energy in terms of mass. But we can write the same equation in the alternative form $\mathrm{m}=\mathrm{E} / \mathrm{c}^{2}$. When expressed in this form, it suggests the possibility of explaining mass in terms of energy. Einstein was aware of this possibility from the beginning. Indeed, his original 1905 paper is entitled, "Does the Inertia of a Body Depend on Its Energy Content?"and it derives $m=E / c^{2}$ not $E=m c^{2}$. Einstein was thinking about fundamental physics, not bombs. 
QED and QCD in Pictures

(FIGURES 1A, 1B, 1C, AND 1D)

The physical content of quantum electrodynamics (QED) is summarized in the algorithm that associates a probability

containing many hubs are not small, and this method is impractical.

At modern particle accelerators, comes to life. For example, in the Large Electron Positron collider (LEP), at the

$1 a$

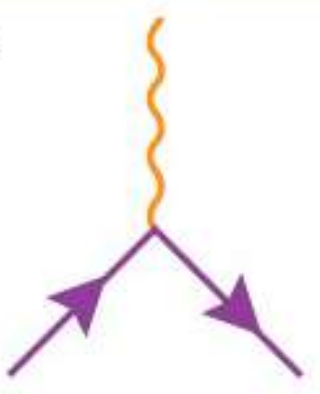

16

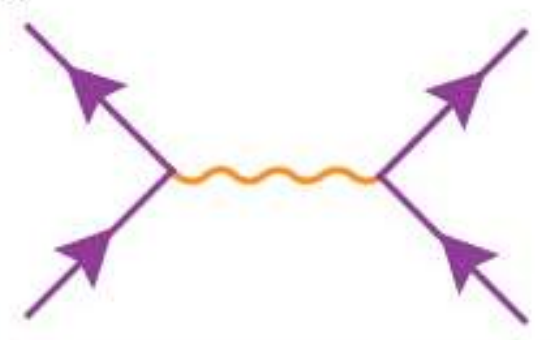
CERN laboratory near Geneva, beams of electrons and antielectrons (positrons) were accelerated to enormous energies.

Powerful, specially designed magnets controlled the paths of the

amplitude with each of its Feynman graphs, depicting a possible process in spacetime.The Feynman graphs are constructed by linking together hubs,more conventionally called interaction vertices, of the form shown in 1a.The solid line depicts the world-line of an electrically charged particle, and the squiggly line straddles the world-line of a photon. By connecting hubs together we can describe physical processes such as the interaction between electrons, as shown in 1b. Quantum chromodynamics (QCD) can be summarized similarly, but with a more elaborate set of ingredients and hubs.

There are three kinds of charges, called colors. Quarks resemble electrons in their mechanical properties (technically, they are spin-1.2 fermions), but their interactions are quite different, because they carry a unit of color charge.Quarks come in several flavors$u, d, s, c, b$, and $t$-so we have $u u u d d d$ and so forth. Only $u$ and $d$,which have very small masses, are important in ordinary matter. The others are heavy and unstable. Gluons resemble photons in their mechanical properties (technically, they are massless spin1 bosons), but their interactions are quite different.There are eight different types of color gluons, which respond to and change the color charges of quarks they interact with. A typical hub for a quark-gluon interaction is shown in 1c, along with a hub for gluon-gluon interaction.The latter has no analog in QED, because the photon carries no electric charge. Asymptotic freedom, and all the drastic differences between how particles with and without color charges are observed to behave, ultimately arise from these new gluon-gluon interactions. In principle we can try to use Feynman diagrams to calculate the quarkquark interaction, as shown in 1d. But unlike in QED, in QCD contributions from graphs particles, and caused

them to circulate in opposite directions around a big storage ring. The paths of these beams intersected at a few interaction regions, where collisions could occur. [After more than a decade of fruitful operation, in which MIT scientists played a leading role, the LEP machine was dismantled in 2000. It is making way for the Large Hadron Collider (LHC), which will use the same tunnel. LHC will collide protons instead of electrons, and will operate at much higher energy. Hence the past tense.] When a collision between a high-energy electron and a highenergy positron occurs, we often observe that manyparticles emerge from the event. [See Figures $2 a$ and $2 b$ on page 29.] The total mass of these particles can be thousands of times the mass of the original electron and positron. Thus mass has been created, physically, from energy.

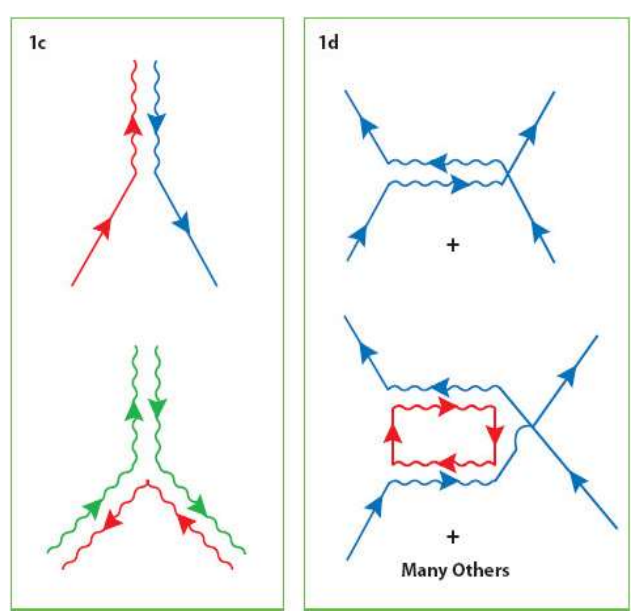




\begin{abstract}
What Matters for Matter
Having convinced ourselves that the question of the origin of mass might make sense, let us now come to grips with it, in the very concrete form that it takes for ordinary matter.

Ordinary matter is made from atoms. The mass of atoms is overwhelmingly concentrated in their nuclei. The surrounding electrons are of course crucial for discussing how atoms interact with each other-and thus for chemistry, biology, and electronics. But they provide less than a part in a thousand of the mass!

Nuclei, which provide the lion's share of mass, are assembled from protons and neutrons. All this is a familiar, well-established story, dating back seventy years or more.

Newer and perhaps less familiar, but by now no less well-established, is the next step: protons and neutrons are made from quarks and gluons. So most of the mass of matter can be traced, ultimately, back to quarks and gluons.
\end{abstract}

\title{
QCD: What It Is
}

The theoryof quarks and gluons is called quantum chromodynamics, or QCD. QCD is a generalization of quantum electrodynamics (QED). For a nice description of quantum electrodynamics, written by an MIT grad who made good, I highly recommend QED: The Strange Theory of Electrons and Light, byRichard Feynman.

The basic concept of QED is the response of photons to electric charge. Figure 1a shows a space-time picture of this core process. Figure $1 \mathrm{~b}$ shows how it can be used to describe the effect of one electric charge on another, through exchange of a "virtual" photon. [A virtual photon is simply one that gets emitted and absorbed without ever having a significant life of its own. So it is not a particle you can observe directly, but it can have effects on things you do observe.] In other words, Figure $1 b$ describes electric and magnetic forces!

Pictures like these, called Feynman diagrams, may look like childish scribbles, but their naïve appearance is misleading. Feynman diagrams are associated with definite mathematical rules that specify how likely it is for the process they depict to occur. The rules for complicated processes, perhaps involving many real and virtual charged particles and many real and virtual photons, are built up in a completely specific and definite way from the core process. It is like making constructions with TinkerToys $\AA$. The particles are different kind of sticks you can use, and the core process provides the hubs that join them. Given these elements, the rules for construction are completely determined. In this way all the contentof Maxwell's equations for radio waves and light, Schrödinger's equation for atoms and chemistry, and Dirac's more refined version including spin-all this, and more, is faithfully encoded in the squiggle [Figure 1a].

At this most primitive level QCD is a lot like QED, but bigger. The diagrams look similar, and the rules for evaluating them are similar, but there are more kinds of sticks and hubs. More precisely, while there is just one kind of charge in QEDnamely, electric charge-QCD has three different kinds of charge. They are called colors, for no good reason. We could label them red, white, and blue; 


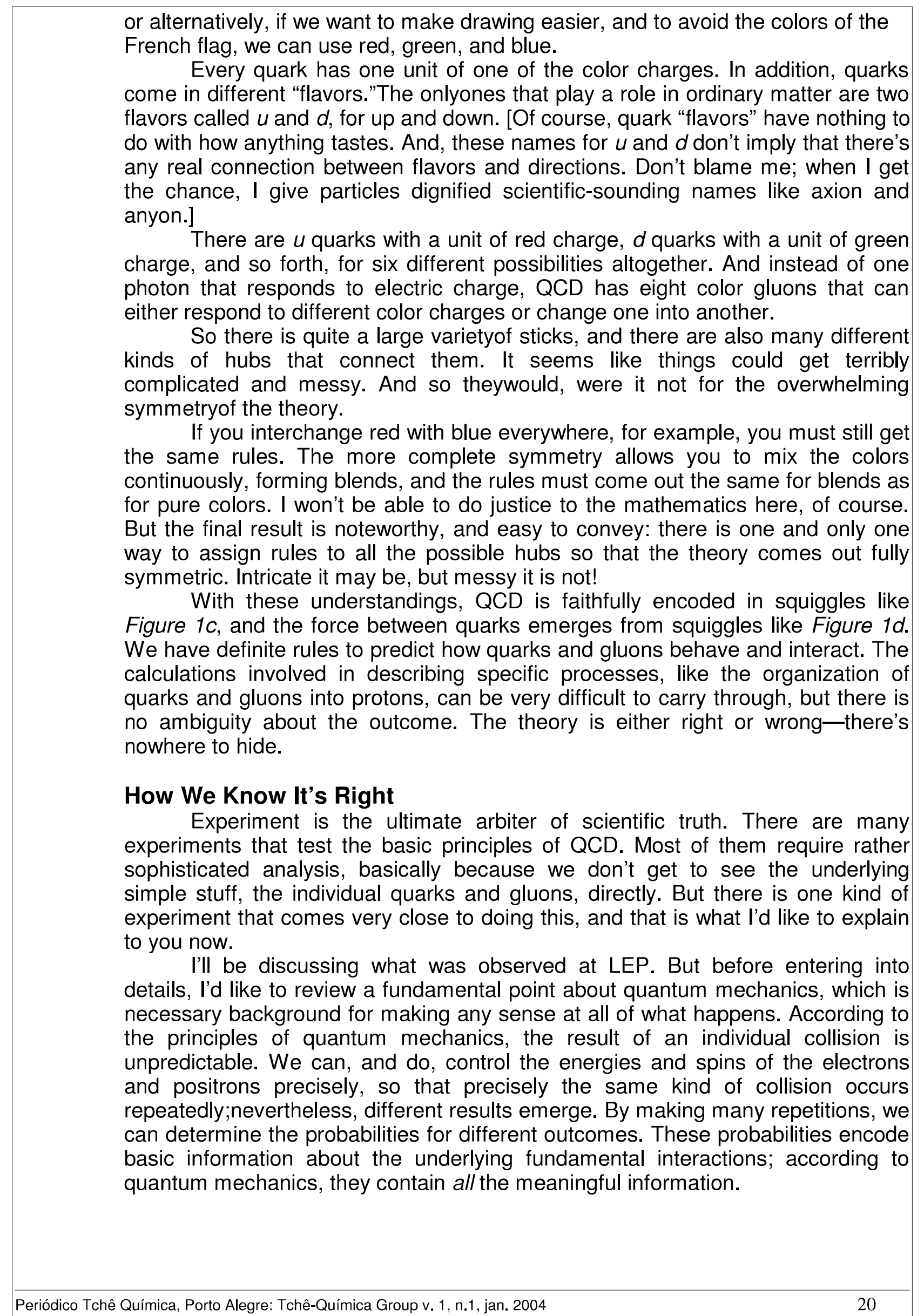


When we examine the results of collisions at LEP, we find there are two broad classes of outcomes. Each happens about half the time.

In one class, the final state consists of a particle and its antiparticle moving rapidly in opposite directions. These could be an electron and an antielectron $\left(\mathrm{e} \cdot \mathrm{e}_{+}\right)$, a muon and an antimuon $(\bullet$ $\left.\bullet_{+}\right)$, or a tau and an antitau $(\bullet \bullet+)$, The little superscripts denote signs of their electric charges, which are all of the same absolute magnitude. These particles, collectively called leptons, are all closely similar in their properties.

Leptons do not carry color charges, so their main interactions are with photons, and thus their behavior should be governed by the rules of QED. This is reflected, first of all, in the simplicityof their final states. Once produced, any of these particles could-in the language of Feynman diagrams-attach a photon using a QED hub, or alternatively, in physical terms, radiate a photon. The basic coupling of photons to a unit charge is fairly weak, however. Therefore each attachment is predicted to decrease the probability of the process being described, and so the most usual case is no attachment.

In fact, the final state $\mathrm{e}^{\cdot} \mathrm{e}^{+} \cdot$, including a photon, does occur, with about $1 \%$ of the rate of simply $e^{*} e^{+}$(and similarly for the other leptons). By studying the

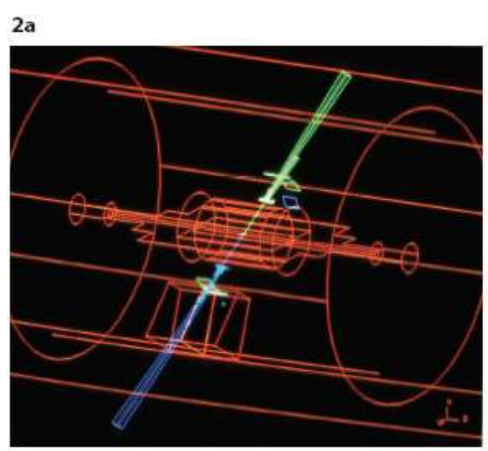

$$
2 b
$$

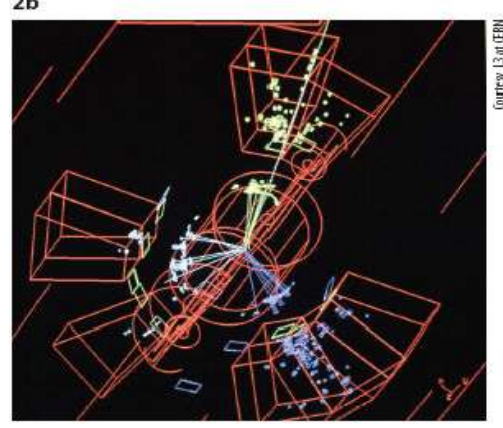

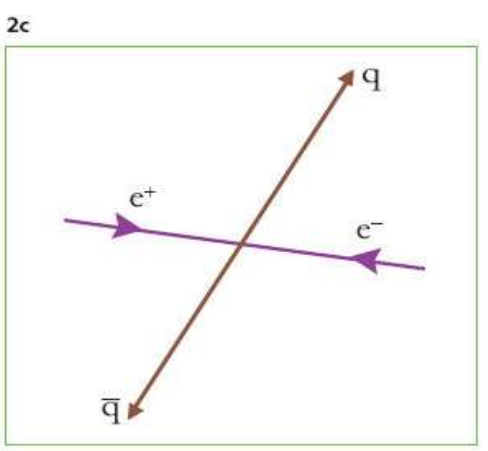

$2 d$

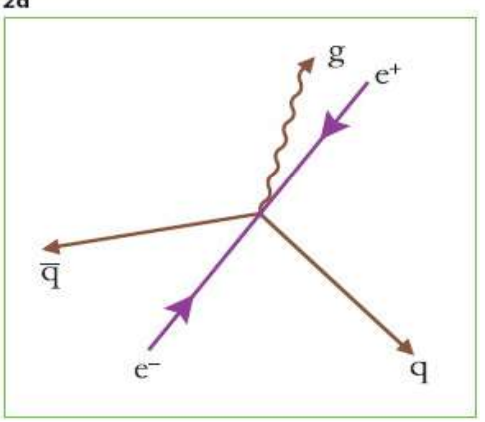

Figures2aand2b:

Real Jets

These are pictures of the results of electron-positron collisions at LEP, taken by the

L3 collaboration led by

Professors Ting,

Becker,andFisher.The alignmentof energetic particles in jets is visible to the naked eye.

Figures2cand2d:

Conceptual Jets These diagramsrepresentour conceptual model of the deep structure beneath jet productionas it is observed.Electrons and positrons annihilate into "pure energy"

(a virtual photon, actually), which materializes into a quark-antiquark pair. The quark and antiquark usually dress themselves with soft radiation, as described in the text,andwe observe a twojet event.

About $10 \%$ of the time, however, a hardgluon is radiated.Then quark,antiquark, and gluon all dress themselves with soft radiation, andwe see three jets.Figures $2 c$ and $2 d$ have been drawn to parallel the geometry of the observations shownin

Figures $2 a$ and 2b.(N.B. To keep things simple, I have not tried to maintain the full color scheme from Figure 1.) 
details of these 3-particle events, such as the probability for the photon to be emitted in different directions (the "antenna pattern") and with different energy, we can check all aspects of our hypothesis for the underlying hub. This provides a wonderfully direct and incisive way to check the soundness of the basic conceptual building block from which we construct QED. We can then go on to address the extremely rare cases $(.01 \%)$ where two photons get radiated, and so forth.

For future reference, let's call this first class of outcomes "QED events."

The other broad class of outcomes contains an entirely different class of particles, and is in manyways far more complicated. In these events the final state typically contains ten or more particles, selected from a menu of pions, rho mesons, protons and antiprotons, and many more. These are all particles that in other circumstances interact stronglywith one another, and they are all constructed from quarks and gluons. Here, they make a smorgasbord of Greek and Latin alphabet soup. It's such a mess that physicists have pretty much given up on trying to describe all the possibilities and their probabilities in detail.

"It's such a mess that physicists have pretty much given up on trying to describe all the possibilities and their probabilities in detail."

Fortunately, however, some simple patterns emerge if we change our focus from the individual particles to the overall flow of energy and momentum.

Most of the time-in about $90 \%$ of the cases - the particles emerge all moving in either one of two possible directions, opposite to one another. We say there are back-to-back jets. (Here, for once, the scientific jargon is both vivid and appropriate.) About $9 \%$ of the time, we find flows in three directions; about .9\% of the time, four directions; and by then we're left with a very small remainder of complicated events that are hard to analyze this way.

l'll call the second broad class of outcomes "QCD events." Representative 2jet and 3-jet QCD events, as they are actually observed, are displayed in Figure 2. Now if you squint a little, you will find that the QED events and the QCD events begin to look quite similar. Indeed, the pattern of energy flow is qualitatively the same in both cases, that is, heavily concentrated in a few narrow jets. There are two main differences. One, relatively trivial, is that multiple jets are more common in QCD than in QED. The other is much more profound. It is that, of course, in the QED events the jets are just single particles, while in the QCD events the jets are sprays of several particles.

In 1973, while I was a graduate student working with David Gross at Princeton, I discovered the explanation of these phenomena. We took the attitude that the deep similarities between the observed basic behaviors of leptons (based on QED) and the strongly interacting particles might indicate that the strongly interacting particles are also ultimately described by a simple, rule-based theory, with sticks and hubs. In other words, we squinted.

To bring our simplified picture of the QCD events into harmonywith the observations, we relied on a theoretical discovery l'll describe momentarily, which we christened asymptotic freedom. (Please notice that our term is not "cute.") Actually, our discovery of asymptotic freedom preceded these specific experiments, so we were able to predict the results of these experiments before theywere performed. 
As a historical matter, we discovered QCD and asymptotic freedom by trying to come to terms with the MIT-SLAC "scaling" experiments done at the Stanford Linear Collider in the late 1960s, for which Jerome Friedman, Henry Kendall, and Richard Taylor won the Nobel Prize in 1990. Since our analysis of the scaling experiments using QCD was (necessarily) more complicated and indirect, l've chosen to focus here on the later, but simpler to understand, experiments involving jets.

The basic concept of asymptotic freedom is that the probability for a fastmoving quark or gluon to radiate away some of its energy in the form of other quarks and gluons depends on whether this radiation is "hard"or "soft". Hard radiation is radiation that involves a substantial deflection of the particle doing the radiating, while soft radiation is radiation that does not cause such a deflection. Thus hard radiation changes the flow of energy and momentum, while soft radiation merely distributes it among additional particles, all moving together. Asymptotic freedom says that hard radiation is rare, but soft radiation is common.

This distinction explains whyon the one hand there are jets, and on the other hand why the jets are not single particles. A QCD event begins as the materialization of quark and antiquark, similar to how a QED event begins as the materialization of leptonantilepton. They usually give us two jets, aligned along the original directions of the quark and antiquark, because only hard radiation can change the overall flow of energy and momentum signifi-cantly, and asymptotic freedom tells us hard radiation is rare.

When a hard radiation does occur, we have an extra jet! But we don't see the original quarks or antiquarks, individually, because they are always accompanied by their soft radiation, which is common.

\section{"Just as for QED, such antenna patterns provide a wonderfully direct and incisive way to check the soundness of the basic conceptual building blocks from which we construct QCD."}

By studying the antenna patterns of the multi-jet QCD events we can check all aspects of our hypotheses for the underlying hubs. Just as for QED, such antenna patterns provide a wonderfully direct and incisive way to check the soundness of the basic conceptual building blocks from which we construct QCD.

Through analysis of this and many other applications, physicists have acquired complete confidence in the fundamental correctness of QCD. By now experimenters use it routinely to design experiments searching for new phenomena, and they refer to what they're doing as "calculating backgrounds" rather than "testing QCD"!

Manychallenges remain, however, to make full use of the theory. The difficulty is always with the soft radiation. Such radiation is emitted very easily, and that makes it difficult to keep track of. You get a vast number of Feynman graphs, each with many attachments, and they get more and more difficult to enumerate, let alone calculate. That's very unfortunate, because when we try to assemble a proton from quarks and gluons none of them can be moving very fast for very long (they're supposed to be inside the proton, after all), so all their interactions involve soft radiation. 


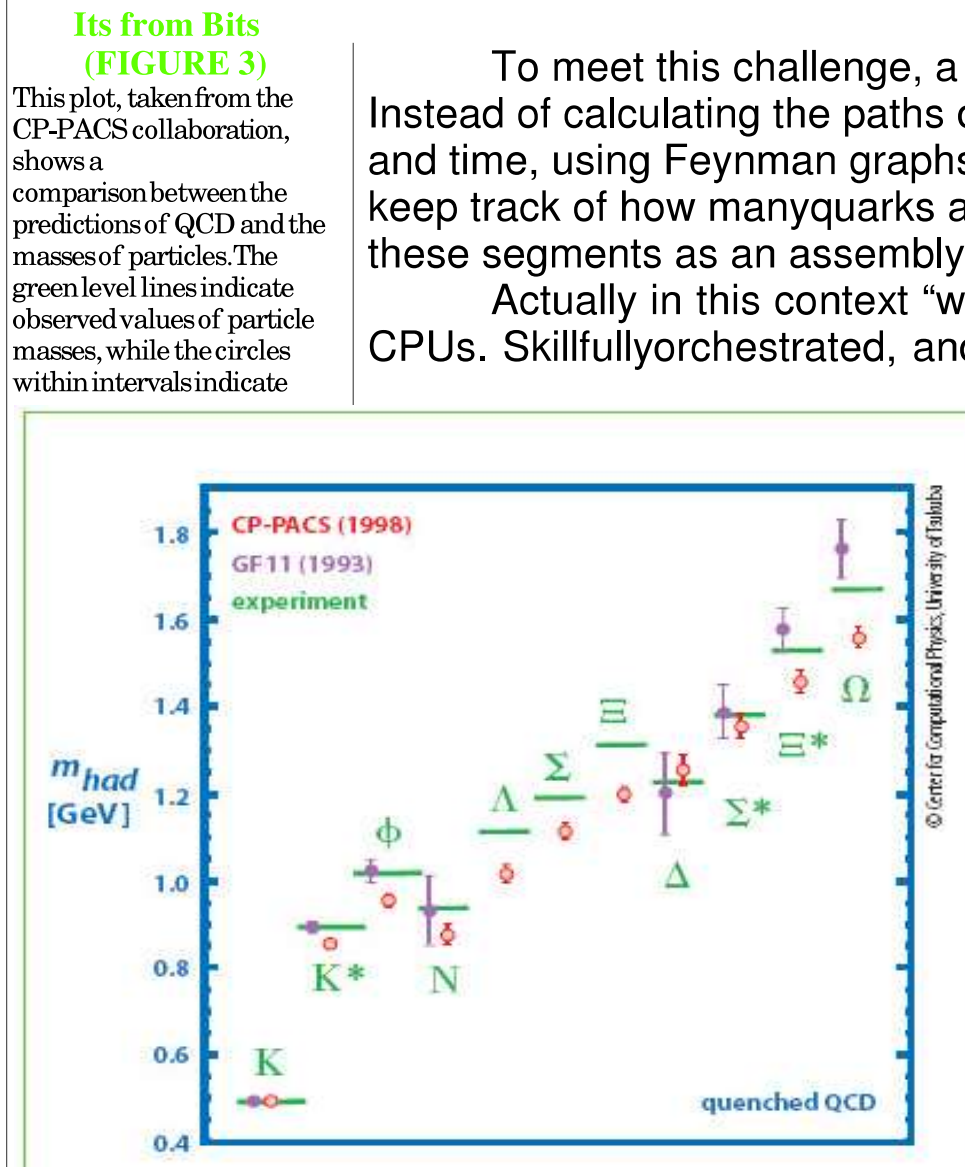

computationalresultsand

theirstatistical

uncertainties. The $\boldsymbol{K}$

meson is theleft-mostentry,

while the proton and neutron

are $N$.The calculations

employ cutting-edge

computertechnology with

massive parallelism, and

even then some

approximations

mustbe introduced to make

the computations

feasible.Theseresultsarea

remarkableembodimentof

the vision thatelementsof

reality can be reproduced by

purely conceptual

constructions-"Its from

Bits"-because the

underlying theory, based on

profoundly symmetrical

equations, contains very few

adjustable parameters. and time, using Feynman graphs, we let each segment of space-time keep track of how manyquarks and gluons it contains. We then treat these segments as an assembly of interacting subsystems.

Actually in this context "we" means a collection of hard-working CPUs. Skillfullyorchestrated, and working full time at teraflop speeds, they manage to produce quite a good account of the masses of protons and other strongly interacting particles, as you can see from Figure 3.

The equations of QCD, which we discovered and proved from very different considerations, survive this extremely intense usage quite well. There's a big worldwide effort, at the frontiers of computer technology and human ingenuity, to do calculations like this more accurately, and to calculate more things.

\section{The Ingredients of QCD, Lite and Full-Bodied}

With the answer in hand, let's examine what we've got. For our purposes it's instructive to compare two versions of QCD, an idealized version I call QCD Lite, and the realistic Full-Bodied version. QCD Lite is cooked up from massless gluons, massless $u$ and $d$ quarks, and nothing else. (Now you can fully appreciate the wit of the name.) If we use this idealization as the basis for our calculation, we get the proton mass low by about $10 \%$.

Full-Bodied QCD differs from QCD Lite in two ways. First, it contains four additional flavors of quarks. These do not appear directly in the proton, but they do have some effect as virtual particles. Second, it allows for non-zero masses of the $u$ and $d$ quarks.

The realistic value of these masses, though, turns out to be small, just a few percent of the proton mass. Each of these corrections changes the predicted mass of the proton by about $5 \%$, as we pass from QCD Lite to Full-Bodied QCD. So we find that $90 \%$ of the proton (and neutron) mass, and therefore $90 \%$ of the mass of ordinary matter, emerges from an idealized theory whose ingredients are entirely massless.

\section{The Origin of (most) Mass}

Now l've shown you the theory that describes quarks and gluons, and therefore has to account for most of the mass of matter. I've described some of the experiments that confirm the theory. 
And l've displayed successful calculations of hadron masses, including the masses of protons and neutrons, using this theory.

In a sense, these calculations settle the question. They tell us the origin of (most) mass. But simply having a computer spit out the answer, after gigantic and totallyopaque calculations, does not satisfyour hunger for understanding. It is particularly unsatisfactory in the present case, because the answer appears to be miraculous.

The computers construct for us massive particles using building blocks-quarks and gluons-that are themselves massless. The equations of QCD Lite output Mass without Mass, which sounds suspiciously like Something for Nothing. How did it happen?

The key, again, is asymptotic freedom. Previously, I discussed this phenomenon in terms of hard and soft radiation. Hard radiation is rare, soft radiation is common. There's another wayof looking at it, mathematically equivalent, that is useful here. From the classical equations of QCD, one would expect a force field between quarks that falls off as the square of the distance, as in ordinary electromagnetism (Coulomb's law). Its enhanced coupling to soft radiation, however, means that when quantum mechanics is taken into account a "bare" color charge, inserted into empty space, will start to surround itself with a cloud of virtual color gluons.

These color gluons fields themselves carry color charge, so they are sources of additional soft radiation. The result is a self-catalyzing enhancement that leads to runaway growth. A small color charge, in isolation, builds up a big color thundercloud.

\section{"But simply having a computer spit out the answer, after gigantic and totally opaque calculations, does not satisfy our hunger for understanding."}

All this structure costs energy, and theoretically the energy for a quark in isolation is infinite. That's whywe never see individual quarks. Having only a finite amount of energy to work with, Nature always finds a way to short-circuit the ultimate thundercloud.

One way is to bring in an antiquark. If the antiquark could be placed right on top of the quark, their color charges would exactly cancel, and the thundercloud would never get started. There's also another more subtle way to cancel the color charge by bringing together three quarks, one of each color.

In practice these exact cancellations can't quite happen, however, because there's a competing effect. Quarks obey the rules of quantum mechanics. It is wrong to think of them simply as tiny particles, rather they are quantum-mechanical wavicles.

They are subject, in particular, to Heisenberg's uncertainty principle, which implies that if you try to pin down their position too precisely, their momentum will be wildly uncertain. To support the possibility of large momentum, they must acquire large energy. In other words, it takes work to pin quarks down. Wavicles want to spread out.

So there's a competition between two effects. To cancel the color charge completely, we'd like to put the quark and antiquark at precisely the same place; but they resist localization, so it's costly to do that.

This competition can result in a number of compromise solutions,together, but where the quark and antiquark (or three quarks) are brought closeare 
perfectly coincident. Their distribution is described by quantum mechanical wave functions. Many different stable wave-patterns are possible, and each corresponds to a different kind of particle that you can observe. There are patterns for protons, neutrons, and for each entry in the whole Greek and Latin smorgasbord. Each pattern has some characteristic energy, because the color fields are not entirely cancelled particles and because the wavicles are somewhat localized. And that, through $\mathrm{m}=\mathrm{Ec}^{2}$, is the origin of mass.

A similar mechanism, though much simpler, works in atoms. Negatively charged electrons feel an attractive electric force from the positively charged nucleus, and from that point of view they'd like to snuggle right on top of it. Electrons are wavicles, though, and that inhibits them. The result, again, is a series of possible compromise solutions.

\section{"The wave patterns that describe protons, neutrons, and their relatives resemble the vibration patterns of musical instruments."}

These are what we observe as the energy levels of the atom. When I give the talk on which this article is based, at this point I use Dean Dauger's marvelous "Atom in a Box" program to show the lovely, almost sensuous patterns of undulating waves that describe the possible states of that simplest of atoms, hydrogen. I hope you will explore "Atom in a Box"for

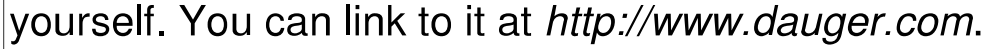

In its absence, I will substitute a classic metaphor. The wave patterns that describe protons, neutrons, and their relatives resemble the vibration patterns of musical instruments. In fact the mathematical equations that govern these superficially very different realms are quite similar.

Musical analogies go back to the prehistory of science. Pythagoras, partly inspired by his discovery that harmonious notes are sounded by strings whose lengths are in simple numerical ratios, proposed that "All things are Number." Kepler spoke of the music of the spheres, and his longing to find their hidden harmonies sustained him through years of tedious calculations and failed guesses before he identified the true patterns of planetary motions.

Einstein, when he learned of Bohr's atomic model, called it "the highest form of musicality in the sphere of thought." Yet Bohr's model, wonderful as it is, appears to us now as a very watered-down version of the true wave-mechanical atom; and the wave-mechanical proton is more intricate and symmetric by far!

I hope that some artist/nerd will rise to the challenge, and construct a "Proton in a Box" for us to play with and admire. 


\section{The World as Concept, Algorithm, and Number}

I will conclude with a few words concerning the broader significance of these developments for our picture of the world.

A major goal of theoretical physics is to describe the world with the greatest possible economy of concepts. For that reason alone, it is an important result that we can largely eliminate mass as an independent property that we are forced to introduce in order to describe matter accurately.

But there is more. The equations that describe the behavior of elementary particles become fundamentally simpler and more symmetric when the mass of the particles is zero. So eliminating mass enables us to bring more symmetry into the mathematical description of Nature.

\section{"Eliminating mass enables us to bring more symmetry into the mathematical description of Nature."}

The understanding of the origin of mass that l've sketched for you here is the most perfect realization we have of Pythagoras' inspiring vision that the world can be built up from concepts, algorithms, and numbers. Mass, a seemingly irreducible property of matter, and a byword for its resistance to change and sluggishness, turns out to reflect a harmonious interplayof symmetry, uncertainty, and energy. Using these concepts, and the algorithms they suggest, pure computation outputs the numerical values of the masses of particles we observe.

Still, as l've already mentioned, our understanding of the origin of mass is by no means complete. We have achieved a beautiful and profound understanding of the origin of most of the mass of ordinary matter, but not of all of it. The value of the electron mass, in particular, remains deeply mysterious even in our most advanced speculations about unification and string theory. And ordinary matter, we have recently learned, supplies only a small fraction of mass in the Universe as a whole. More beautiful and profound revelations surely await discovery. We continue to search for concepts and theories that will allow us to understand the origin of mass in all its forms, by unveiling more of Nature's hidden symmetries.

Frank Wilczek is considered one of the world's most eminent theoretical physicists. He is known, among other things, for the discovery of asymptotic freedom, the development of quantum chromodynamics, the invention of axions, and the discovery and exploitation of new forms of quantum statistics (anyons).

When only 21 years old and a graduate student at Princeton University, in work with David Gross he defined the properties of color gluons, which hold atomic nuclei together. Presently his main obsessions are exotic superfluidities on the one hand and dark energy on the other. He suspects the two are connected. Professor Wilczek received his B.S. degree from the University of Chicago and his Ph.D. from Princeton University. He taught at Princeton from 1974 to 1981. During the period 1981 to 1988 , he was the Chancellor Robert Huttenback Professor of Physics at the University of California at Santa Barbara, and the first permanent member of the National Science Foundation's Institute for Theoretical Physics. In the fall of 2000, he moved from the Institute for Advanced Study, where he was the J.R. Oppenheimer Professor, to the Massachusetts Institute of Technology, where he is the Herman Feshbach Professor of Physics. He has been a Sloan Foundation Fellow (1975-77) and a MacArthur Foundation Fellow (1982-87). He has received UNESCO's Dirac Medal, the American Physical Society's Sakurai Prize, the Michelson Prize from Case Western University, and the Lorentz Medal of the Netherlands Academy for his contributions to the development of theoretical physics, and the Lilienfeld Prize for his writing.

$\mathrm{He}$ is a member of the National Academy of Sciences, the Netherlands Academy of Sciences, and the American Academy of Arts and Sciences. He is a Trustee of the University of Chicago, and an official 


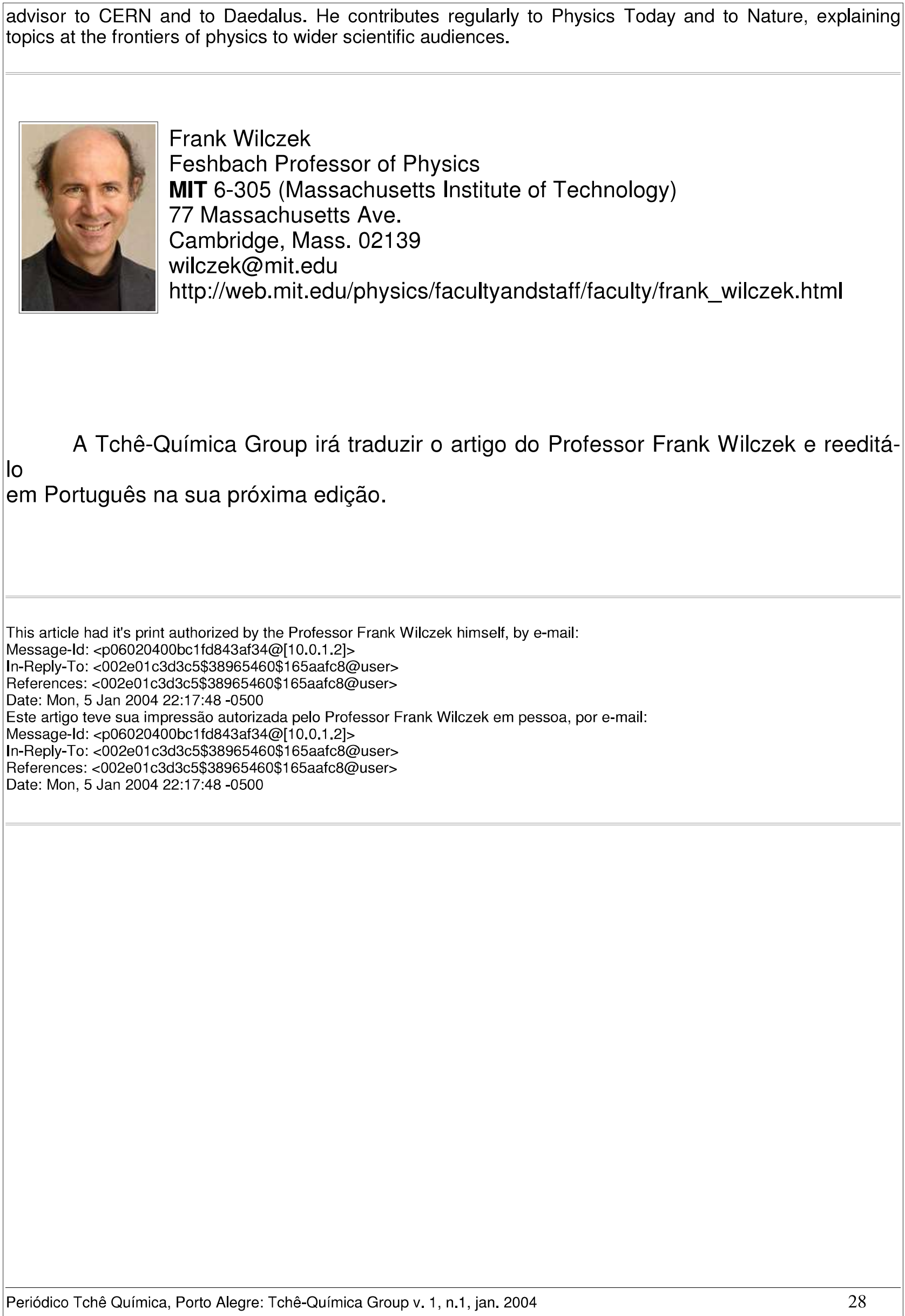

Periódico Tchê Química, Porto Alegre: Tchê-Química Group v. 1, n.1, jan. 2004
Tchê Quimica (ISSN: 1806-0374; 2179-0302) is an open-access journal since 2004. Journal DOI: 10.52571/PTQ. http://www.tchequimica.com. The Periódico Tchê Química (ISSN: 1806-0374; 2179-0302) is an open
This text was introduced in this file in 2021 for compliance reasons. (c) The Author(s)

OPEN ACCESS. This article is licensed under a Creative Commons Attribution 4.0 (CC BY 4.0) International License, which permits use, sharing, adaptation, distribution, and reproduction in any medium or format, as long as you give appropriate credit to the original author(s) and the source, provide a link to the Creative Commons license, and indicate if changes were made. The images or other third-party material in this article are included in the article 's Creative Commons license unless indicated otherwise in a credit line to the material. If material is not included in the article's Creative Commons license and your intended use is not permitted by statutory regulation or exceeds the permitted use, you will need to obtain permission directly from the copyright holder. To view a copy of this license, visit http://creativecommons.org/licenses/by/4.0/ 